\title{
The Stability Ratio for a Dispersion of Particles Covered by an Ion-Penetrable Charged Membrane
}

\author{
JYH-PING Hsu ${ }^{1}$ AND Yung-CHIH KuO \\ Department of Chemical Engineering, National Taiwan University, Taipei, Taiwan 10617, Republic of China
}

Received January 17, 1996; accepted March 28, 1996

\begin{abstract}
The electrostatic interaction between two particles covered by an ion-penetrable charged membrane suspended in an $a: b$ electrolyte solution is estimated. A perturbation method is employed to solve the governing nonlinear Poisson- Boltzmann equation. The approximate analytical expression derived for potential distribution yields a sufficiently accurate estimate. Both the stability ratio and the critical coagulation concentration of the counterions of the system under consideration are derived. The results of a numerical simulation reveal that the stability ratio decreases with an increase in the size of a particle and increases with the total amount of fixed charges in the membrane phase. For a constant total amount of fixed charges and particle size, the stability ratio increases with a decrease in the thickness of the membrane. We show that monodispersed particles are more stable than polydispersed particles. (C) 1996 Academic Press, Inc.

Key Words: stability ratio, spherical particles; critical coagulation concentration, counterions; Poisson-Boltzmann equation, nonlinear; membrane, ion-penetrable, charged; electrical potential distribution, approximate, analytical.
\end{abstract}

\section{INTRODUCTION}

One of the key characteristics of a dispersed colloidal suspension is its stability ratio, defined as (rate of fast coagulation $) /($ rate of slow coagulation ). The numerator denotes the rate of coagulation in the absence of an interaction energy barrier, and the denominator is that in the presence of an interaction energy barrier. Here, the interaction energy includes the electrostatic repulsive energy and the van der Waals attractive energy (1). The estimation of the former requires knowledge about the electrostatic potential distribution between two charged surfaces, which is governed by the Poisson-Boltzmann equation (PBE). This equation in its general form is nonlinear. Although there exists some fundamental inconsistency in the PBE which arises from the origin of the corresponding Poisson equation, the deviation from the exact value is tolerable for conditions often encoun-

\footnotetext{
${ }^{1}$ To whom correspondence should be addressed. E-mail: T8504009@ CCMS.NTU.EDU.TW.
}

tered in practice (2). The solution to a general PBE is nontrivial, except for limited cases. The degree of difficulty depends largely on the geometry of a charged surface, the types of electrolyte in the liquid phase, and the boundary conditions on the surface. In practice, either drastic assumptions are made and a simplified PBE is solved, or it is solved numerically.

Previous efforts on the estimation of the stability ratio of a dispersed system were mainly based on rigid surfaces (e.g., $3-5)$. Although this model is appropriate for most of the inorganic colloids, it can be unrealistic for some of the dispersed entities in practice. These include biocolloids and particles covered by an artificial membrane (6-11). A typical example of the former is human erythrocytes, the peripheral zone of which contain a glycoprotein layer about $15 \mathrm{~nm}$ thick. This zone possesses some ionogenic groups and forms the outer boundary of the lipid layer $(12,13)$. An example of the latter is dispersed entities in polymer-induced flocculation. Here, an entity comprises a rigid core and a layer of adsorbed polymer molecules, which often bear fixed charges. In an analysis of the interaction between an ion-penetrable particle and a rigid particle, Terui et al. (14) derived expressions for the interaction potential and the electrostatic interaction force between particles. The analysis was extended to various combinations of two types of particles, and expressions for critical coagulation concentration of counterions were derived (15). These analyses $(14,15)$ were based on a low level of surface potential, symmetric electrolyte, and uniformly distributed fixed charges in the surface layer of a particle.

In a recent study the electrostatic interactions between spherical particles coated with an ion-penetrable charged membrane immersed in an electrolyte solution at a low electrostatic potential were analyzed (16). The result obtained was adopted to estimate both the stability ratio and the critical coagulation concentration of counterions. If the potential is low, the governing PBE can be approximated by a linear expression, which is readily solvable. In the present study, the linear model of (16) is extended to a general case in which both the level of potential and the type of electrolyte are arbitrary. 


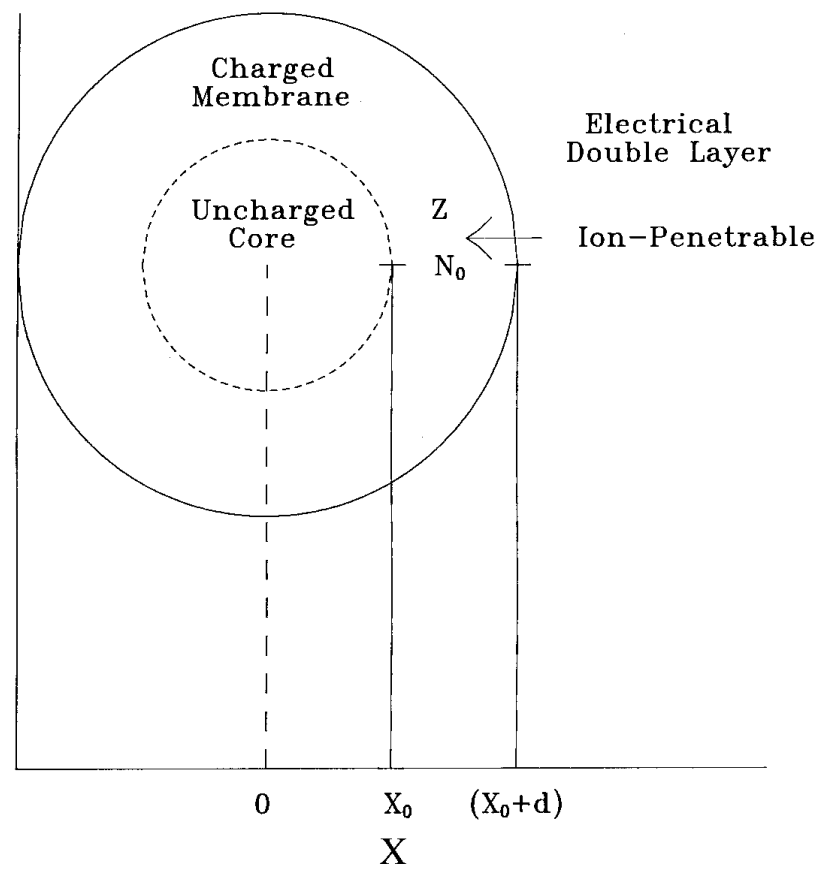

FIG. 1. A schematic representation of the structure of a particle under consideration. $Z$ and $N_{0}$ are, respectively, the valence and the density of the charged groups. The region $X>\left(X_{0}+d\right)$ represents the liquid phase, $d$ being the dimensionless thickness of the membrane. The region $X<X_{0}$ denotes the rigid core of a particle; $X_{0}<X<\left(X_{0}+d\right)$ is the ion-penetrable membrane phase.

\section{MODELING}

By referring to Fig. 1, we consider a spherical particle composed of a rigid core and an ion-penetrable, charged membrane immersed in an $a: b$ electrolyte solution. Denote the dimensionless sizes of the rigid core and the membrane as $X_{0}$ and $d$, respectively. Here, $X_{0}=\kappa r_{0}$, where $r_{0}$ and $\kappa$ are the size of the rigid core and the reciprocal Debye length, respectively. Without loss of generality, we assume that the fixed charges in the membrane are negative and distributed uniformly.

The electrical potential distribution in spherical geometry is described by the PBE

$$
\frac{d^{2} \psi}{d X^{2}}+\frac{2}{X} \frac{d \psi}{d X}=\frac{g+i N}{a+b}, \quad i=0,1,
$$

where $\psi=e \phi / k_{\mathrm{B}} T, g=[\exp (b \psi)-\exp (-a \psi)], X=\kappa r$, $\kappa^{2}=e^{2} a(a+b) n_{a}^{0} / \epsilon_{0} \epsilon_{\mathrm{r}} k_{\mathrm{B}} T$, and $N=Z N_{\mathrm{A}} N_{0} / a n_{a}^{0}$. In these expressions, $\phi$ denotes the electrical potential; $e$ is the elementary charge; $N_{0}$ and $Z$ are, respectively, the density and the valence of the charged groups in the membrane $\left(-Z e N_{\mathrm{A}} N_{0}\right.$ is the density of fixed charges ); $N_{\mathrm{A}}$ represents the Avogadro number; $\epsilon_{\mathrm{r}}$ and $\epsilon_{0}$ are the relative permittivities of the solution and the vacuum, respectively; $n_{a}^{0}$ is the number concentration of cations in the bulk liquid phase; $k_{\mathrm{B}}$ and
$T$ are, respectively, the Boltzmann constant and the absolute temperature; $i$ represents a region index ( $i=0$ denotes the double-layer region, $i=1$ the membrane phase); and $r$ is the distance measured from the center of a particle. The boundary conditions associated with [1] are assumed to be

$$
\begin{gathered}
\psi \rightarrow 0 \text { and }(d \psi / d X) \rightarrow 0 \quad \text { as } X \rightarrow \infty \\
\left.\psi\right|_{x \rightarrow\left(x_{0}+d\right)^{-}}=\left.\psi\right|_{x \rightarrow\left(x_{0}+d\right)^{+}}=\psi_{\mathrm{d}} \\
\left.(d \psi / d X)\right|_{x \rightarrow\left(x_{0}+d\right)^{-}}=\left.(d \psi / d X)\right|_{x \rightarrow\left(x_{0}+d\right)^{+}} \\
\psi \rightarrow \psi_{\mathrm{c}} \text { and }(d \psi / d X) \rightarrow 0 \quad \text { as } X \rightarrow X_{0} .
\end{gathered}
$$

In these expressions, $\psi_{\mathrm{c}}$ and $\psi_{\mathrm{d}}$ are, respectively, the dimensionless electrical potential at the outer boundary of the rigid core of a particle and that at the membrane-liquid interface. Suppose that $\psi$ can be expanded in the perturbation series (17)

$$
\begin{aligned}
\psi & =\sum_{v=0}^{\infty} \frac{h_{v}}{X^{v}} \\
& \cong h_{0}+\left(\frac{h_{1}}{X}\right)+\left(\frac{h_{2}}{X^{2}}\right)
\end{aligned}
$$

Substituting [2] into [1] with $i=0$ and solving the resultant expression give the electrical potential distribution for the double-layer region (Appendix A),

$$
\begin{aligned}
& h_{0}=\left(\frac{2}{a}\right) \ln \left\{\begin{array}{l}
1+\left[\tanh \left(a \psi_{\mathrm{d}} / 4\right)\right] \\
\frac{\times \exp \left[-k_{3}\left(X-X_{0}-d\right)\right]}{1-\left[\tanh \left(a \psi_{\mathrm{d}} / 4\right)\right]} \\
\times \exp \left[-k_{3}\left(X-X_{0}-d\right)\right]
\end{array}\right\} \\
& h_{1}=\left(\frac{1}{a k_{3}}\right) \sinh \left(\frac{a h_{0}}{2}\right)\left[\tanh ^{2}\left(\frac{a \psi_{\mathrm{d}}}{4}\right)\right. \\
& \left.-\tanh ^{2}\left(\frac{a h_{0}}{4}\right)-2 \ln \left[\frac{\tanh \left(a \psi_{\mathrm{d}} / 4\right)}{\tanh \left(a h_{0} / 4\right)}\right]\right] \\
& h_{2}=X\left(h_{0}\right) \frac{a \sinh \left(a h_{0} / 2\right)}{8 k_{3}}\left[u \int_{0}^{h_{0}} \frac{h_{1}^{2} \sinh \left(a h_{0} / 2\right)}{X\left(h_{0}\right)} d h_{0}\right. \\
& \left.+\int_{h_{0}}^{\psi_{0}} \frac{u h_{1}^{2} \sinh \left(a h_{0} / 2\right)}{X\left(h_{0}\right)} d h_{0}\right]
\end{aligned}
$$

where $k_{3}, u$, and $X\left(h_{0}\right)$ are defined in Appendix A.

If the membrane is sufficiently thick, the dimensionless Donnan potential $\psi_{\text {Don }}$ and $\psi_{\mathrm{d}}$ are related by (Appendix A) 


$$
\begin{aligned}
& \left(\frac{2}{a+b}\right)^{1 / 2}\left\{\frac{1}{b}\left[\exp \left(b \psi_{\mathrm{d}}\right)-1\right]+\frac{1}{a}\left[\exp \left(-a \psi_{\mathrm{d}}\right)-1\right]\right\}^{1 / 2} \\
& -\frac{1}{X_{0}+d}\left[\frac{4}{a} \tanh \left(\frac{a \psi_{\mathrm{d}}}{4}\right)\right]+\frac{4}{\left(X_{0}+d\right)^{2}} \\
& \times\left[\frac{\tanh ^{2}\left(a \psi_{\mathrm{d}} / 4\right)-2 \ln \left[\cosh \left(a \psi_{\mathrm{d}} / 4\right)\right]}{k_{3} \sinh \left(a \psi_{\mathrm{d}} / 2\right)}\right] \\
& =\left(\frac{2}{a+b}\right)^{1 / 2}\left\{\frac{1}{b}\left[\exp \left(b \psi_{\mathrm{d}}\right)-\exp \left(b \psi_{\text {Don }}\right)\right]\right. \\
& +\frac{1}{a}\left[\exp \left(-a \psi_{\mathrm{d}}\right)-\exp \left(-a \psi_{\text {Don }}\right)\right] \\
& \left.+N\left(\psi_{\mathrm{d}}-\psi_{\text {Don }}\right)\right\}^{1 / 2}-\frac{4}{X_{0}+d} \\
& \quad \times\left\{\frac{1}{a} \tanh \left[\frac{a\left(\psi_{\mathrm{d}}-\psi_{\text {Don }}\right)}{4}\right]\right\}+\frac{4}{\left(X_{0}+d\right)^{2}}
\end{aligned}
$$$$
\times\left\{\frac{\tanh ^{2}\left[a\left(\psi_{\mathrm{d}}-\psi_{\text {Don }}\right) / 4\right]}{k_{3} \sinh \left[a\left(\psi_{\mathrm{d}}-\psi_{\text {Don }}\right) / 2\right]} .\right.
$$

The electrostatic interaction energy between two particles $I_{\mathrm{el}}(R)$ can be estimated by substituting [2] - [5] into [B16] (see Appendix B) to yield

$$
\begin{aligned}
I_{\mathrm{el}}(R)=\frac{16 \pi}{a k_{3}^{3} \kappa^{3} R} \sum_{n=1}^{2}\left\{P _ { n , n ^ { \prime } } \sum _ { t = 1 } ^ { 3 } \left\{A _ { t } \operatorname { e x p } \left[k_{3}(2 t-1)\right.\right.\right. \\
\\
\left.\left.\left.\times\left(X_{0, n}+d_{n}-R\right)\right]\right\}\right\}, \quad n \neq n^{\prime},
\end{aligned}
$$

where the subscripts $n$ and $n^{\prime}$ denote particles $n$ and $n^{\prime}$, respectively, $d_{n}$ is the dimensionless thickness of the membrane phase of particle $n$, and $R$ is the dimensionless centerto-center distance between two particles (Fig. 2). The values of $A_{1}, A_{2}, A_{3}$, and $P_{n, n^{\prime}}$ are defined by

$$
\begin{aligned}
A_{1}= & \left(3+\alpha_{1}\right)\left(X_{0, n}+d_{n}\right) \cosh \left[k_{3}\left(X_{0, n}+d_{n}\right)\right] \\
& -\left(3+\alpha_{1}\right) X_{0, n} \cosh \left(k_{3} X_{0, n}\right)-\left(\frac{3}{k_{3}}+\alpha_{1}\right) \\
& \times\left(X_{0, n}+d_{n}\right) \sinh \left[k_{3}\left(X_{0, n}+d_{n}\right)\right] \\
& +\left(\frac{3}{k_{3}}+\alpha_{1}\right) \times X_{0, n} \sinh \left(k_{3} X_{0, n}\right)
\end{aligned}
$$

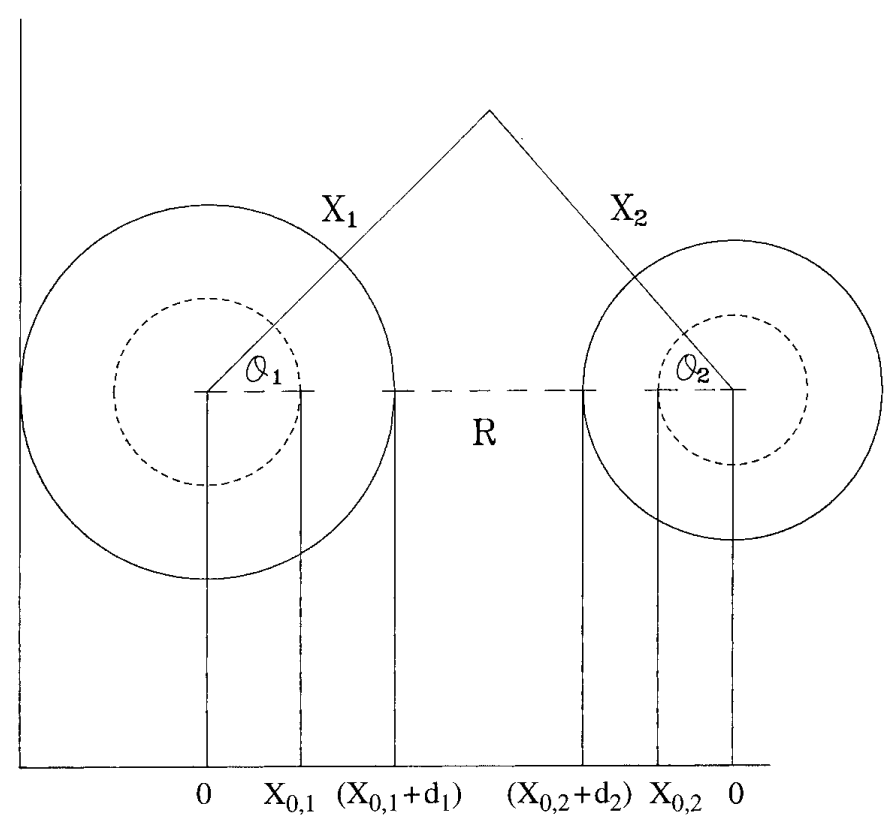

FIG. 2. Schematic representation of a system containing two particles.

$$
\begin{aligned}
A_{2}= & -\left[\frac{4}{27 k_{3}}+\frac{2}{9 \alpha_{4}}\left(\alpha_{2}^{2}-2 \alpha_{2}^{2} \alpha_{3}\right)\right] \tanh ^{2}\left(\frac{a \psi_{\mathrm{d}, n}}{4}\right) \\
& \times\left\{3 k_{3}\left(X_{0, n}+d_{n}\right) \cosh \left[3 k_{3}\left(X_{0, n}+d_{n}\right)\right]\right. \\
& -3 k_{3} X_{0, n} \cosh \left(3 k_{3} X_{0, n}\right) \\
& \left.-\sinh \left[3 k_{3}\left(X_{0, n}+d_{n}\right)\right]+\sinh \left(3 k_{3} X_{0, n}\right)\right\} \\
A_{3}= & {\left[\frac{3}{32 \alpha_{4}}\left(2 \alpha_{2}^{2} \alpha_{3}-\alpha_{2}^{2}-\alpha_{2}\right)\right] \tanh ^{4}\left(\frac{\mathrm{a} \psi_{\mathrm{d}, n}}{4}\right) } \\
& \times\left\{5 k_{3}\left(X_{0, n}+d_{n}\right) \cosh \left[5 k_{3}\left(X_{0, n}+d_{n}\right)\right]\right. \\
& -5 k_{3} X_{0, n} \cosh \left(5 k_{3} X_{0, n}\right) \\
& \left.-\sinh \left[5 k_{3}\left(X_{0, n}+d_{n}\right)\right]+\sinh \left(5 k_{3} X_{0, n}\right)\right\}
\end{aligned}
$$

$\alpha_{1}=\left(\frac{1}{2}\right) \tanh ^{2}\left(\frac{a \psi_{\mathrm{d}, n}}{4}\right)+k_{3}\left(X_{0, n}+d_{n}\right)-3+\frac{\alpha_{5}}{\alpha_{4}}$

$$
\alpha_{2}=\tanh ^{2}\left(\frac{a \psi_{\mathrm{d}, n}}{4}\right)+\ln \left\{\frac{1}{2}\left[1+\cosh \left(\frac{a \psi_{\mathrm{d}, n}}{2}\right)\right]\right\}
$$

$$
\begin{aligned}
\alpha_{3}=\frac{\operatorname{coth}\left(a \psi_{\mathrm{d}, n} / 2\right)}{\sinh \left(a \psi_{\mathrm{d}, n} / 2\right)} & -\frac{1}{2} \\
& \times \ln \left\{\frac{1}{2}\left[1+\cosh \left(\frac{a \psi_{\mathrm{d}, n}}{2}\right)\right]\right\} \\
\alpha_{4} & =600 k_{3}^{4}\left(X_{0, n}+d_{n}\right)
\end{aligned}
$$




$$
\begin{aligned}
\alpha_{5}=\alpha_{2}^{2} \alpha_{3} \sinh ^{2}\left(\frac{a \psi_{\mathrm{d}, n}}{4}\right) & \\
+ & {\left[2 \alpha_{2}^{2} \alpha_{3}+\frac{1}{2} \alpha_{2}^{2}+\alpha_{2}\right] \times \sinh ^{4}\left(\frac{a \psi_{\mathrm{d}, n}}{4}\right) } \\
P_{n, n^{\prime}}= & L_{n}\left[\tanh \left(\frac{a \psi_{\mathrm{d}, n^{\prime}}}{4}\right)\right]
\end{aligned}
$$

shown that the value of $R$ at which $I_{\mathrm{t}}$ has its primary maximum, $R_{\max }$, is

$$
\begin{aligned}
R_{\max } \cong\left[\beta_{6}+\left(\beta_{7}^{3}\right.\right. & \left.\left.+\beta_{6}^{2}\right)^{1 / 2}\right]^{1 / 3} \\
& +\left[\beta_{6}-\left(\beta_{7}^{3}+\beta_{6}^{2}\right)^{1 / 2}\right]^{1 / 3}-\frac{\beta_{3}}{3},
\end{aligned}
$$

where

$$
\times \exp \left[k_{3}\left(X_{0, n^{\prime}}+d_{n^{\prime}}-X_{0, n}-d_{n}\right)\right]
$$

where the energy density $L_{n}$ is defined in Appendix B.

The electrostatic interaction force between two particles, $f_{\mathrm{el}}(R)$, can be calculated by

$$
\begin{aligned}
f_{\mathrm{el}}(R)= & -\kappa\left(\frac{d I_{\mathrm{el}}}{d R}\right) \\
= & \frac{\kappa I_{\mathrm{el}}}{R}+\frac{16 \pi}{a k_{3}^{2} \kappa^{2} R} \sum_{n=1}^{2}\left\{P _ { n , n ^ { \prime } } \sum _ { t = 1 } ^ { 3 } \left\{A_{t}(2 t-1)\right.\right. \\
& \left.\left.\times \exp \left[k_{3}(2 t-1)\left(X_{0, n}+d_{n}-R\right)\right]\right\}\right\} \\
= & \frac{\kappa\left(k_{3} R+1\right)}{R} I_{\mathrm{el}}+\frac{32 \pi}{a k_{3}^{2} \kappa^{2} R} \\
& \times \sum_{n=1}^{2}\left\{P _ { n , n ^ { \prime } } \sum _ { t = 2 } ^ { 3 } \left\{A_{t}(t-1)\right.\right. \\
& \left.\left.\times \exp \left[k_{3}(2 t-1)\left(X_{0, n}+d_{n}-R\right)\right]\right\}\right\},
\end{aligned}
$$$$
n \neq n^{\prime} \text {. }
$$

Note that if $R \rightarrow \infty$, both $I_{\mathrm{el}}$ and $f_{\mathrm{el}}$ vanish, as expected.

The stability ratio can be regarded as the reciprocal collision efficiency which leads to coagulation. It can be measured by the ratio of particle fluxes without and with an energy barrier (1). Therefore the kinetic property can be described by the interaction energy between two particles. According to Fuchs (18) and Overbeek (19), the stability ratio of the system under consideration, $W$, can be estimated by

$$
W=\int_{1}^{\infty}\left(\frac{1}{R_{\mathrm{r}}^{2}}\right) \exp \left(\frac{I_{\mathrm{t}}}{k_{\mathrm{B}} T}\right) d R_{\mathrm{r}}
$$

where $R_{\mathrm{r}}=R / D_{2}$, and $I_{\mathrm{t}}$ is the total interaction energy between two particles. The latter includes the electrostatic repulsion energy and the van der Waals attraction energy. On the basis of [7], [8], [C2e], [C3], and [C5a], it can be

$$
\beta_{3}=\beta_{2}-2 D_{2}-\frac{A_{132}}{12 D_{3} \beta_{1}}
$$

$\beta_{6}=\frac{\left(9 \beta_{3} \beta_{4}-27 \beta_{5}-2 \beta_{3}\right)}{54}$

$\beta_{7}=\frac{\left(3 \beta_{3}-\beta_{3}^{2}\right)}{9}$

$\beta_{5}=D_{2}^{2} \beta_{2}$

$\beta_{4}=D_{2}\left(D_{2}-2 \beta_{2}\right)$

$\beta_{2}=\frac{16 \pi}{a k_{3}^{3} \kappa^{3} \beta_{1}} \sum_{n=1}^{2}\left\{P_{n, n^{\prime}} \sum_{t=1}^{3}\left\{A_{t} \times \exp \left[k_{3}(2 t-1)\right.\right.\right.$

$\left.\left.\left.\times\left(X_{0, n^{\prime}}+d_{n^{\prime}}\right)\right]\right\}\right\}, \quad n \neq n^{\prime}$

$$
\begin{aligned}
\beta_{1}= & \frac{16 \pi}{a k_{3}^{2} \kappa^{3}} \sum_{n=1}^{2}\left\{P _ { n , n ^ { \prime } } \sum _ { t = 1 } ^ { 3 } \left\{A_{t}(2 t-1)\right.\right. \\
& \left.\left.\times \exp \left[k_{3}(2 t-1)\left(X_{0, n^{\prime}}+d_{n^{\prime}}\right)\right]\right\}\right\}, \\
& \\
& n \neq n^{\prime} .
\end{aligned}
$$

In these expressions, $A_{132}$ denotes the Hamaker constant, and $\mathrm{D}_{2}$ and $\mathrm{D}_{3}$ are defined in [C2c] and [C2b], respectively ( see Appendix C). Substituting [7], [C1], [C2e], [C5a], and [10] into [C4] yields

$$
W \cong \exp \left\{\left[I_{\mathrm{el}}\left(R_{\max }\right)-\frac{A_{132} D_{3}}{12\left(R_{\max }-D_{2}\right)}\right] / k_{\mathrm{B}} T\right\} .
$$

The critical coagulation concentration (CCC) can be determined by

$$
\begin{gathered}
I_{\mathrm{el}}\left(R_{\mathrm{c}}\right)-\frac{A_{132} D_{3}}{12\left(R_{\mathrm{c}}-D_{2}\right)}=0 \\
f_{\mathrm{el}}\left(R_{\mathrm{c}}\right)+\frac{\kappa A_{132} D_{3}}{12\left(R_{\mathrm{c}}-D_{2}\right)^{2}}=0
\end{gathered}
$$

where $R_{\mathrm{c}}$ is the dimensionless center-to-center distance between two particles at the CCC. Solving these equations yields 
TABLE 1

Average Deviation $Q(\%)$ in the Potential Distribution of the Present Perturbation Method, $\psi$, from the Exact $\mathbf{N}$ umerical $\mathrm{C}$ alculation, $\psi^{* a}$

\begin{tabular}{cccc}
\hline & \multicolumn{3}{c}{$N_{0}(M)$} \\
\cline { 2 - 4 }$X_{0}$ & $10^{-3}$ & $5 \times 10^{-3}$ & $10^{-2}$ \\
\hline 1 & 3.77 & 4.84 & 5.61 \\
2 & 3.13 & 4.27 & 4.85 \\
4 & 2.32 & 3.50 & 4.39 \\
6 & 2.17 & 3.28 & 3.96 \\
\hline
\end{tabular}

${ }^{a}$ For the case $Z=1, d=X_{0} / 4$, ionic strength $=10^{-3} M, \epsilon_{\mathrm{r}}=78$, and $T=298.15 \mathrm{~K} . t$ equally spaced points in the range $\left[X_{0}+d, X_{0}+d+3\right]$ are chosen, and the potentials at these points are evaluated. $Q$ is defined by $100 \%\left[\Sigma_{a=1}^{3} \Sigma_{b=1}^{3} \Sigma_{j=1}^{t} \mid\left(\psi^{*}-\psi\right) / \psi^{*}\right] / 9 t$, where $a$ and $b$ are the valences of the cation and anion, respectively.

$$
R_{\mathrm{c}} \cong \gamma+\left[\gamma\left(\gamma+D_{2}\right)\right]^{1 / 2}
$$

where

$$
\gamma=D_{2}-\frac{A_{132} D_{3}}{12 \beta_{1}}
$$

The CCC for counterions can be estimated by substituting [14] into either [12] or [13].

\section{DISCUSSION}

Table 1 shows the average percentage deviation in the potential distribution estimated by [2] from the exact values, estimated by solving [1] numerically. As can be seen from this table, the performance of the present perturbation method is satisfactory.

Figure 3 illustrates the simulated variation in the stability ratio $W$ as a function of the linear size of the rigid core of a particle at various densities of fixed charges in the membrane phase. This figure suggests that $W$ decreases with increases of $X_{0}$. This is consistent with the classic DLVO theory together with the Fuchs model (1), and the result for the case of low potential (16). For a fixed $X_{0}$, the greater the density of fixed charges $N_{0}$, the greater the $W$. This is expected, since the greater the $N_{0}$, the greater the electrostatic repulsive force between two particles.

Figure 4 shows the effect of membrane thickness on the stability ratio at a constant total amount of fixed charges and particle size. As can be seen from Fig. 4, the stability ratio increases with decreases in the membrane thickness. This is because at a constant total amount of fixed charges, the smaller the thickness of membrane, the more concentrated the fixed charges, and the greater the electrostatic repulsion energy. Figure 4 also suggests that the stability ratio increases with the total amount of fixed charges, as expected.

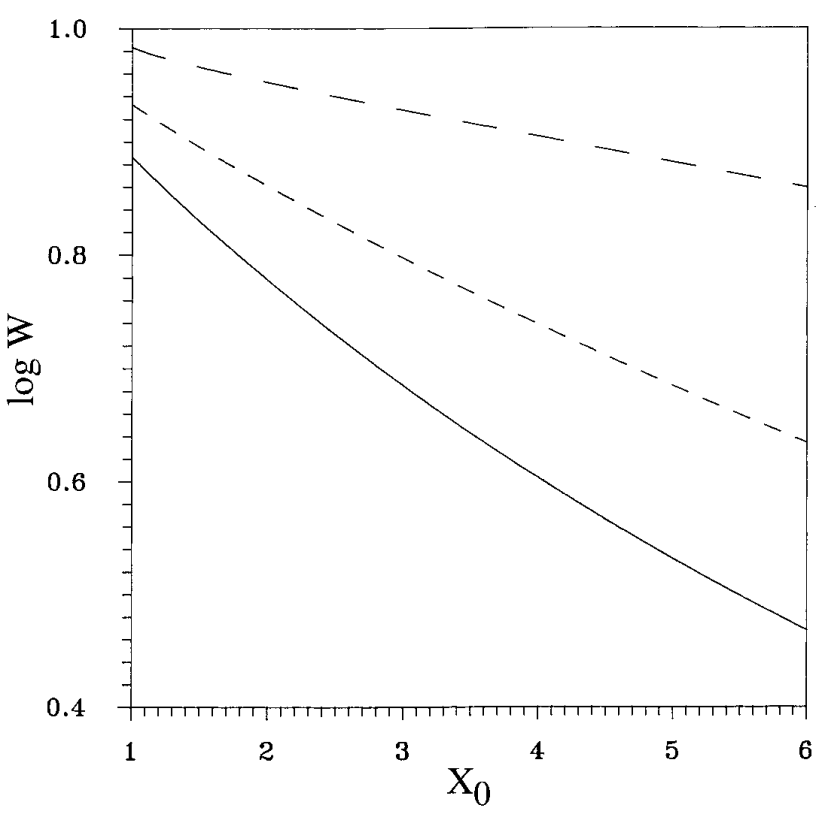

FIG. 3. Variation of $\log W$ as a function of the radius of the rigid core of a particle for the case $X_{0,1}=X_{0,2}=X_{0}, d_{1}=d_{2}=X_{0} / 4$. - , $N_{0}=10^{-3} \mathrm{M}$; ---, $N_{0}=5 \times 10^{-3} M$; - - - , $N_{0}=10^{-2} M$. Key: $\epsilon_{\mathrm{r}}=78, T=298.15 \mathrm{~K}$, $Z_{1}=Z_{2}=1, a=2, b=1, A_{132}=10^{-20} \mathrm{~J}$, and ionic strength $=10^{-3} \mathrm{M}$.

Figure 5 illustrates the variation in the stability ratio as a function of the size ratio of two interacting particles, $\left(X_{0,1}\right.$ $\left.+d_{1}\right) /\left(X_{0,2}+d_{2}\right)$, at a fixed mean size of two particles, $\left[\left(X_{0,1}\right.\right.$ $\left.\left.+d_{1}\right)\left(X_{0,2}+d_{2}\right)\right]^{1 / 2}$. This figure shows that the smaller the size ratio (the greater the difference in the sizes of two

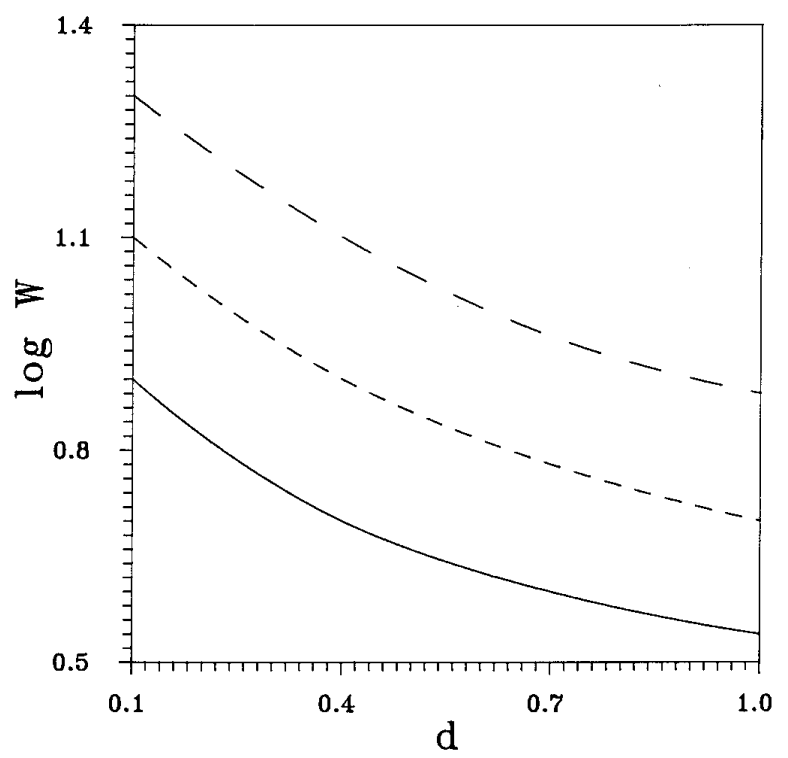

FIG . 4. Variation of $\log W$ as a function of the membrane thickness at a constant total amount of fixed charges, TC, and particle size for the case $d_{1}=d_{2}=d, X_{0,1}+d_{1}=X_{0,2}+d_{2}=5 .-, \mathrm{TC}=2.26 \times 10^{-22} \mathrm{~mol}$; ,--- TC $=1.13 \times 10^{-21} \mathrm{~mol} ;---$, TC $=2.26 \times 10^{-21}$ mol. Key: see the legend to Fig. 3 . 


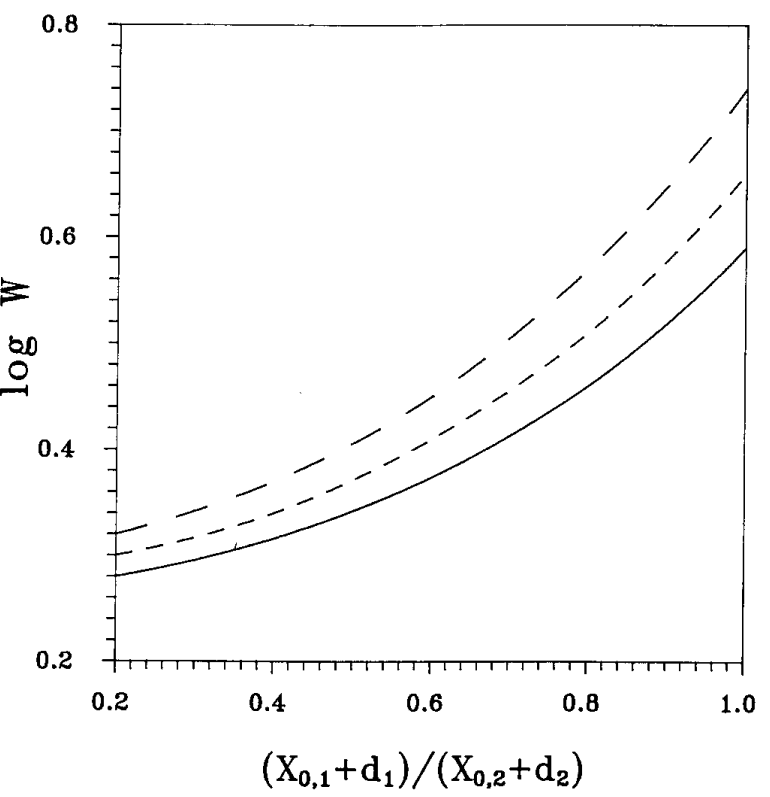

FIG. 5. Variation of $\log W$ as a function of the relative size of two interacting particles at a fixed mean size of two particles $\bar{a}$ for the case $d_{1}$ $=d_{2}=1$ and $N_{0}=8 \times 10^{-3} M .-, \bar{a}=11 ;---, \bar{a}=13 ;---, \bar{a}=$ 15. Key: see the legend to Fig. 3 .

particles), the smaller the stability ratio. In other words, monodispersed particles are more stable than polydispersed particles. This can be deduced from Eqs. [C2]-[C2d]. The smaller the size ratio, the smaller the value of $D_{4}$, the greater the absolute van der Waals attraction energy, and the smaller the stability ratio.

Under certain conditions, the results of the present study can be either simplified or reduced to those reported in the literature. We consider five special cases.

\subsection{Two Identical Particles}

If the interacting particles are identical, then $X_{0,1}=X_{0,2}$ $=X_{0}, d_{1}=d_{2}=d, \psi_{\mathrm{d}, 1}=\psi_{\mathrm{d}, 2}=\psi_{\mathrm{d}}, P_{1,2}=P_{2,1}=P\left(X_{0}\right.$, $\left.d, \psi_{\mathrm{d}}\right), A_{t}=A_{t}\left(X_{0}, d, \psi_{\mathrm{d}}\right), t=1,2,3$, and [7] gives

$$
\begin{aligned}
& I_{\mathrm{el}}(H)= \frac{32 \pi}{a k_{3}^{3} \kappa^{3} R} P\left(X_{0}, d, \psi_{\mathrm{d}}\right) \\
& \times \sum_{t=1}^{3}\left\{A_{t}\left(X_{0}, d, \psi_{\mathrm{d}}\right)\right. \\
&\left.\quad \quad \times \exp \left[k_{3}(2 t-1)\left(X_{0}+d-R\right)\right]\right\} .
\end{aligned}
$$

\subsection{A Spherical Particle and a Flat Surface}

If particle 1 is spherical and particle 2 becomes a flat surface, [7] reduces to

$$
\begin{aligned}
I_{\mathrm{el}}(H)=\frac{24 \pi}{a k_{3}^{4} \kappa^{3}} L_{1}[ & \left.\tanh \left(\frac{a \psi_{\mathrm{d}, 2}}{4}\right)\right]\left[1-\exp \left(-k_{3} d_{2}\right)\right] \\
& \times \exp \left[k_{3}\left(X_{0,1}+d_{1}-D_{3} H\right)\right],
\end{aligned}
$$

where $H$ is the dimensionless surface-to-surface distance between the particle and the surface.

\subsection{Two Parallel Flat Surfaces}

In the case of two parallel flat surfaces, it can be shown that

$$
\begin{aligned}
I_{\mathrm{el}}(H)= & \frac{8 \pi}{a k_{3}^{4} \kappa^{3}} \sum_{n=1}^{2}\left\{L_{n}\left[\tanh \left(\frac{a \psi_{\mathrm{d}, n}}{4}\right)\right]\right. \\
& \times\left\{3\left[1-\exp \left(-k_{3} d_{n}\right)\right] \exp \left(-k_{3} D_{3} H\right)\right. \\
& -\frac{4}{27}\left(3 k_{3}-1\right)\left[1-\exp \left(-3 k_{3} d_{n}\right)\right] \\
& \left.\left.\times\left[\tanh \left(\frac{a \psi_{\mathrm{d}, n}}{4}\right)\right] \exp \left(-3 k_{3} D_{3} H\right)\right\}\right\} .
\end{aligned}
$$

\subsection{Rigid Particles and 1:1 Electrolytes}

For 1:1 electrolytes and rigid particles, [3]-[5] reduce to those derived by Dukhin et al. (20).

\subsection{Single Particle}

Suppose that the radius of one of the two interacting particles approaches zero, i.e., $\left(X_{0, n}+d_{n}\right) \rightarrow 0, n=1$ or 2 . Then $d_{n} \rightarrow 0$ and $\psi_{n}$ does not exist. In this case, [7] implies that $I_{\mathrm{el}}(R) \rightarrow 0$ and $I_{\mathrm{vdw}} \rightarrow 0$ by [B2].

\section{APPENDIX A}

Substituting [2] into [1] and collecting terms of the same order in $X$, we obtain the potential distribution in the doublelayer region,

$$
\begin{gathered}
\frac{d^{2} h_{0}}{d X^{2}}=\frac{g_{0}+i N}{a+b} \\
\frac{d^{2} h_{1}}{d X^{2}}-\frac{1}{a+b}\left[b \exp \left(b h_{0}\right)\right. \\
\left.+a \exp \left(-a h_{0}\right)\right] h_{1}=\frac{-2 d h_{0}}{d X}
\end{gathered}
$$

$$
\begin{aligned}
\frac{d^{2} h_{2}}{d X^{2}} & -\frac{2}{X} \frac{d h_{2}}{d X}+\frac{2}{X^{2}} h_{2} \\
- & \frac{1}{a+b}\left[b \exp \left(b h_{0}\right)+a \exp \left(-a h_{0}\right)\right] h_{2} \\
& =\frac{1}{2(a+b)}\left[b^{2} \exp \left(b h_{0}\right)-a^{2} \exp \left(-a h_{0}\right)\right] h_{1}^{2},
\end{aligned}
$$


where $g_{0}=g\left(h_{0}\right)$. The boundary conditions associated with these equations are

$$
h_{v} \text { and }\left(d h_{v} / d X\right) \rightarrow 0 \text { as } X \rightarrow \infty, \quad v=0,1,2, \ldots
$$

$h_{0} \rightarrow \psi_{\mathrm{d}}, h_{v} \rightarrow 0$ as $X \rightarrow\left(X_{0}+d\right), \quad v=1,2, \ldots$

Solving [A1] with $i=0$ subject to [A4] and [A5] gives [3] with

$$
k_{3}= \begin{cases}{\left[(k-2) k_{1}+2 k_{2}\right] / k,} & \text { if } k \leqslant 4 \\ {\left[2 k_{1}+(k-2) k_{2}\right] / k,} & \text { if } k>4,\end{cases}
$$

where $k_{1}=2 /\left\{k^{1 / 2}\left[(k / 2)^{2 /(k-2)}-1\right]\right\}, k_{2}=2 / k^{1 / 2}$, and $k$ $=2+2 b / a$ (17). Similarly, solving [A2] and [A3] subject to [A4] and [A5] gives [4] and [5] with

$$
\begin{aligned}
& u=\frac{\cosh \left(a \psi_{\mathrm{d}} / 2\right)}{\sinh ^{2}\left(a \psi_{\mathrm{d}} / 2\right)}-\frac{\cosh \left(a h_{0} / 2\right)}{\sinh ^{2}\left(a h_{0} / 2\right)} \\
&-\ln \left[\frac{\tanh \left(a h_{0} / 4\right)}{\tanh \left(a \psi_{\mathrm{d}} / 4\right)}\right]
\end{aligned}
$$

$$
\begin{array}{r}
X\left(h_{0}\right) \\
=\frac{1}{2 k_{3}} \ln \left[\left(\frac{\cosh \left(a h_{0} / 2\right)+1}{\cosh \left(a h_{0} / 2\right)-1}\right)\left(\frac{\cosh \left(a \psi_{\mathrm{d}} / 2\right)-1}{\cosh \left(a \psi_{\mathrm{d}} / 2\right)+1}\right)\right] \\
+X_{0}+d . \quad[\text { A } 8]
\end{array}
$$

Substituting [3]-[5] into [2] gives the potential distribution in the double-layer region. For 1:1 electrolytes, [3][5] reduce to the results proposed by Dukhin et al. (20).

Differentiating [2] with respect to $X$ and applying [A5], we obtain

$$
\begin{aligned}
\left(\frac{d \psi}{d X}\right)_{X_{0}+d} & =\sum_{v=0}^{\infty}\left[\left(\frac{d h_{v}}{d X}\right) / X^{v}\right]_{X_{0}+d} \\
\cong\left(\frac{d h_{0}}{d X}\right)_{X_{0}+d} & +\frac{1}{X_{0}+d}\left(\frac{d h_{1}}{d X}\right)_{X_{0}+d} \\
& +\frac{1}{\left(X_{0}+d\right)^{2}}\left(\frac{d h_{2}}{d X}\right)_{X_{0}+d} .
\end{aligned}
$$

Integrating [A1] $-[\mathrm{A} 3]$ with $i=0$, subject to [A4] and employing [A5], we have

$$
\begin{array}{r}
\left(\frac{d h_{0}}{d X}\right)_{\left(X_{0}+d\right)^{+}}=\left[\frac{2}{a+b}\right]^{1 / 2}\left\{\frac{1}{b}\left[\exp \left(b \psi_{\mathrm{d}}\right)-1\right]\right. \\
\left.+\frac{1}{a}\left[\exp \left(-a \psi_{\mathrm{d}}\right)-1\right]\right\}^{1 / 2}
\end{array}
$$

$$
\begin{aligned}
& \left(\frac{d h_{1}}{d X}\right)_{\left(X_{0}+d\right)^{+}}=-\frac{4}{a} \tanh \left(\frac{a \psi_{\mathrm{d}}}{4}\right) \\
& \left(\frac{d h_{2}}{d X}\right)_{\left(X_{0}+d\right)^{+}}=\frac{-8 \tanh ^{2}\left(a \psi_{\mathrm{d}} / 4\right)}{k_{3} \sinh \left(a \psi_{\mathrm{d} / 2}\right)} .
\end{aligned}
$$

Equation [1d] can be rewritten in terms of $h_{v}$ as

$$
\begin{aligned}
\left(\frac{d h_{0}}{d X}\right) \rightarrow 0,\left(\frac{d h_{v}}{d X}\right) \rightarrow 0, h_{0} \rightarrow \psi_{\mathrm{c}}, \text { and } h_{v} \rightarrow 0 & \\
& \text { as } X \rightarrow X_{0}, v=1,2, \ldots
\end{aligned}
$$

Integrating [A1] $-[\mathrm{A} 3]$ with $i=1$ subject to [A13] and applying [A5], we obtain

$$
\begin{aligned}
\left(\frac{d h_{0}}{d X}\right)_{\left(X_{0}+d\right)^{-}}= & {\left[\frac{2}{a+b}\right]^{1 / 2}\left\{\frac{1}{b}\left[\exp \left(b \psi_{\mathrm{d}}\right)-\exp \left(b \psi_{\mathrm{c}}\right)\right]\right.} \\
& +\frac{1}{a}\left[\exp \left(-a \psi_{\mathrm{d}}\right)-\exp \left(-a \psi_{\mathrm{c}}\right)\right] \\
\left.+N\left(\psi_{\mathrm{d}}-\psi_{\mathrm{c}}\right)\right\}^{1 / 2} & {[\mathrm{~A} 14] } \\
\left(\frac{d h_{1}}{d X}\right)_{\left(X_{0}+d\right)^{-}}= & -\frac{4}{a} \tanh \left(\frac{a\left(\psi_{\mathrm{d}}-\psi_{\mathrm{c}}\right)}{4}\right) \\
\left(\frac{d h_{2}}{d X}\right)_{\left(X_{0}+d\right)^{-}}= & \frac{-8 \tanh \left[\cosh \left(a\left(\psi_{\mathrm{d}}-\psi_{\mathrm{c}}\right) / 4\right)\right]}{k_{3} \sinh \left(a\left(\psi_{\mathrm{d}}-\psi_{\mathrm{c}}\right) / 2\right)} .
\end{aligned}
$$

If the membrane phase is sufficiently thick, there is no net charge as $X \rightarrow X_{0}$, and $\psi$ approaches $\psi_{\text {Don }}$, the dimensionless Donnan potential. In this case, letting $i=1$ in [1] yields

$$
g\left(\psi_{\text {Don }}\right)+N=0 .
$$

$\psi_{\text {Don }}$ can be estimated by solving this equation. On the basis of [1b], [A9], [A10]-[A12], and [A14]-[A16], [6] can be recovered.

\section{APPENDIX B}

Let $\mathbf{E}$ be the strength of electric field. We have

$$
\nabla \cdot(\phi \mathbf{E})=\phi \nabla \cdot \mathbf{E}+\mathbf{E} \cdot \nabla \phi
$$

where $\nabla$ is the differential operator. Since $\mathbf{E}=-\nabla \phi$, the PBE can be written as 


$$
\begin{aligned}
\nabla \cdot \mathbf{E} & =-\nabla \cdot \nabla \phi \\
& =\frac{-\left[\sum_{v=1}^{M} z_{v} e n_{v}^{0} \exp \left(-z_{v} \psi\right)-i \rho_{\mathrm{fix}}\right]}{\epsilon_{0} \epsilon_{\mathrm{r}}},
\end{aligned}
$$

where $n_{v}^{0}$ and $z_{v}$ denote, respectively, the number concentration of the $v$ th ion species in the bulk liquid phase and its valence, $M$ is the number of ion species, and $\rho_{\text {fix }}$ represents the density of fixed charges evaluated by

$$
\rho_{\text {fix }}=Z e N_{\mathrm{A}} N_{0} \text {. }
$$

For the present system, the density of internal energy, $u_{\mathrm{el}}$, is identical to the electric field energy, i.e.,

$$
u_{\mathrm{el}}=\left(\frac{\epsilon_{0} \epsilon_{\mathrm{r}}}{2}\right) \mathbf{E} \cdot \mathbf{E} \text {. }
$$

For dilute solutions the density of entropy, $s_{\mathrm{el}}$, is

$$
s_{\mathrm{el}}=-\left(\frac{k_{\mathrm{B}}}{2}\right) \sum_{v=1}^{M} n_{v} \ln \left(\frac{n_{v}}{n_{v}^{0}}\right),
$$

where $n_{v}$ is the number concentration of species $v$. According to the Gibbs-Helmholtz equation, we have

$$
f_{\mathrm{el}}=u_{\mathrm{el}}-T s_{\mathrm{el}},
$$

where $f_{\mathrm{el}}$ is the density of electrical free energy. Substituting [B3] and [B4] into this expression, employing the Boltzmann distribution law for ions, and using [B1] and [B2], we have

$$
f_{\mathrm{el}}=-\left(\frac{\epsilon_{0} \epsilon_{\mathrm{r}}}{2}\right) \nabla \cdot(\phi \mathbf{E})-\frac{i \rho_{\mathrm{fix}} \phi}{2} .
$$

Denote the volume of the membrane phase and that of the liquid phase as $V_{1}$ and $V_{2}$, respectively, and let $V=V_{1}+$ $V_{2}$. The total free energy of the system, $F_{\text {el }}^{\mathrm{t}}$, is

$$
F_{\mathrm{el}}^{\mathrm{t}}=\int f_{\mathrm{el}} d V
$$

Substituting [B6] into [B7] gives

$$
F_{\mathrm{el}}^{\mathrm{t}}=-\left(\frac{\epsilon_{0} \epsilon_{\mathrm{r}}}{2}\right) \int_{V} \nabla \cdot(\phi \mathbf{E}) d V-\frac{1}{2} \int_{V_{1}} \rho_{\mathrm{fix}} \phi d V_{1},
$$

where $d V$ and $d V_{1}$ represent volume elements. Applying the Gauss divergence theorem yields

$$
F_{\mathrm{el}}^{\mathrm{t}}=-\left(\frac{\epsilon_{0} \epsilon_{\mathrm{r}}}{2}\right) \int_{A} \phi \mathbf{E} \cdot \mathbf{n} d A-\frac{1}{2} \int_{V 1 \rho \mathrm{fix}} \phi d V_{1},
$$

where $\mathbf{n}$ denotes the unit outer normal vector of surface element $d A$. Since both $\mathbf{E}$ and $\phi$ are continuous, and $\phi \mathbf{E}$ is bounded, the surface integral in [B9] vanishes, and we have

$$
F_{\mathrm{el}}^{\mathrm{t}}=-\frac{1}{2} \int_{V_{1}} \rho_{\mathrm{fix}} \phi d V_{1} \text {. }
$$

For a rigid surface $\mathbf{E}$ is not continuous at the solid-liquid interface. Thus, if $\mathbf{n}$ is parallel to $\mathbf{E}$, letting $V_{1}=0$ in [B9] leads to

$$
F_{\mathrm{el}}^{\mathrm{t}}=\frac{\sigma \phi_{0} A}{2}
$$

where $\phi_{0}$ denotes the surface potential, and $A$ is the area of the surface.

The electrostatic energy of a system containing $K$ particles, $I_{\mathrm{el}}(\underline{R})$, can be calculated by

$$
I_{\mathrm{el}}(\underline{R})=F_{\mathrm{el}}^{\mathrm{t}}(\underline{R})-F_{\mathrm{el}}^{\mathrm{t}}(\infty),
$$

where $R$ is the set of distances $R_{n, n^{\prime}}, n, n^{\prime}=1,2, \ldots, K, n$ $\neq n^{\prime}, \bar{R}_{n, n^{\prime}}$ being the dimensionless center-to-center distance between particle $n$ and $n^{\prime}$, with $R_{n, n^{\prime}}=R_{n^{\prime}, n}$. Suppose that the double layer around a particle is thin. Employing [B10] and $[\mathrm{B} 2 \mathrm{a}]$, we obtain

$$
\begin{aligned}
& F_{\mathrm{el}}^{\mathrm{t}}(\underline{R})=\sum_{n=1}^{K} \int_{V_{1, n}} L_{n} \psi d V_{1, n} \\
& F_{\mathrm{el}}^{\mathrm{t}}(\infty)=\sum_{n=1}^{K} \int_{V_{1, n}} L_{n} \psi_{n, i=1} d V_{1, n}
\end{aligned}
$$

$$
\psi=\left\{\begin{array}{r}
\psi_{n, i=1}+\sum_{n^{\prime}} \psi_{n^{\prime}, i=0}, \quad X_{0, n}<X_{n}<\left(X_{0, n}+d_{n}\right), \\
n, n^{\prime}=1,2, \ldots, K, n^{\prime} \neq n \\
\psi_{n, i=0}+\sum_{n^{\prime}} \psi_{n^{\prime}, i=0},\left(X_{0, n}+d_{n}\right)<X_{n}, \\
\left(X_{0, n^{\prime}}+d_{n^{\prime}}\right)<X_{n^{\prime}}, \quad n, n^{\prime}=1,2, \ldots, K, \\
n^{\prime} \neq n,
\end{array}\right.
$$

where $L_{n}=-z_{n} N_{\mathrm{A}} N_{0}, n k_{B} T / 2, d V_{1, n}=2 \pi X_{n}^{2} \sin \theta_{n} d X_{n} d \theta_{n} /$ $\kappa^{3}$, and $\theta_{n}$ is the angle between line segments defined by $R_{n, n^{\prime}}$ and $X_{n} . F_{\mathrm{el}}^{\mathrm{t}}(\infty)$ is the electrostatic free energy for the case any pair of two particles are infinitly apart. Since $\psi_{n, i=1}$ is a function of $X_{n}, F_{\mathrm{el}}^{\mathrm{t}}(\infty)$ is independent of $R$. For a system containing two particles, [B14] reduces to 


$$
\psi=\left\{\begin{array}{lr}
\psi_{1, i=1}+\psi_{2, i=0}, & X_{0,1}<X_{1}<\left(X_{0,1}+d_{1}\right) \\
\psi_{1, i=0}+\psi_{2, i=0}, & \left(X_{0,1}+d_{1}\right)<X_{1}, \\
\psi_{1, i=0}+\psi_{2, i=1}, & \left(X_{0,2}+d_{2}\right)<X_{2} \\
& X_{0,2}<X_{2}<\left(X_{0,2}+d_{2}\right) .
\end{array}\right.
$$

By referring to Fig. 2, we have

$$
\begin{aligned}
& X_{1}=\left(R^{2}+X_{2}^{2}-2 R X_{2} \cos \theta_{2}\right)^{1 / 2} \\
& X_{2}=\left(R^{2}+X_{1}^{2}-2 R X_{1} \cos \theta_{1}\right)^{1 / 2}
\end{aligned}
$$

where $R$ denotes the dimensionless center-to-center distance between two particles, and the angles $\theta_{1}$ and $\theta_{2}$ are defined in Fig. 2. For a two-particle system, [B12] becomes

$$
I_{\mathrm{el}}(R)=\sum_{n=1}^{2} \int_{V_{1, n^{\prime}}} L_{n} \psi_{n^{\prime}, i=0} d V_{1, n^{\prime}}, \quad n^{\prime} \neq n .
$$

\section{APPENDIX C}

The total interaction energy $I_{\mathrm{t}}$ includes the van der Waals energy $I_{\mathrm{vdw}}$ and the electrostatic energy $I_{\mathrm{el}}$, i.e.,

$$
I_{\mathrm{t}}=I_{\mathrm{vdw}}+I_{\mathrm{el}}
$$

We assume that (1)

$$
\begin{array}{r}
I_{\mathrm{vdw}}=\frac{-A_{132}}{12 H}\left[\frac{1}{1+D_{4} H}+\frac{H}{1+H+D_{4} H^{2}}\right. \\
\left.+2 H \ln \left(\frac{H+D_{4} H^{2}}{1+H+D_{4} H^{2}}\right)\right],
\end{array}
$$

where

$$
\begin{aligned}
D_{4} & =\frac{D_{3}}{2 D_{2}} \\
D_{3} & =\frac{D_{1}}{D_{2}} \\
D_{2} & =X_{0,1}+d_{1}+X_{0,2}+d_{2} \\
D_{1} & =2\left(X_{0,1}+d_{1}\right)\left(X_{0,2}+d_{2}\right) \\
H & =\frac{\left(R-D_{2}\right)}{D_{3}},
\end{aligned}
$$

where $A_{132}$ denotes the Hamaker constant and $D_{3}$ represents the dimensionless reduced radius. The interaction force between particles, $f_{t}$, can be evaluated by

$$
\begin{aligned}
f_{\mathrm{t}} & =-\left(\frac{\kappa}{D_{3}}\right)\left(\frac{d I_{\mathrm{t}}}{d H}\right) \\
& =f_{\mathrm{el}}-\left(\frac{\kappa}{D_{3}}\right)\left(\frac{d I_{\mathrm{vdw}}}{d H}\right) .
\end{aligned}
$$

The stability ratio of the system, $W$, can be estimated by [9]. As an approximation, the total interaction energy is replaced with its primary maximum, $I_{\mathrm{t}, \max }$, and the integration procedure suggested by Overbeek (19) leads to

$$
W \cong \exp \left(\frac{I_{\mathrm{t}, \max }}{k_{\mathrm{B}} T}\right) .
$$

As $H \rightarrow 0,[\mathrm{C} 2]$ reduces to

$$
\begin{aligned}
I_{\mathrm{vdw}} & =\frac{-A_{132}}{12 H}\left[1+H\left(1-D_{4}\right)+2 H \ln H+\cdots\right] \\
& \cong \frac{-A_{132}}{12 H}
\end{aligned}
$$

If the size of one of the two interacting particles approaches infinity, i.e., $X_{0, n} \rightarrow \infty, n=1$ or 2 , [C2] reduces to

$$
I_{\mathrm{vdw}}=\frac{-A_{132}}{6}\left[\frac{1+2 H}{2 H(1+H)}+\ln \left(\frac{H}{1+H}\right)\right] \text {. }
$$

If the sizes of two interacting particles approach infinity, the problem reduces to the interaction of two parallel planar surfaces. In this case, [C2] gives

$$
I_{\mathrm{vdw}}=-A_{132} A_{\mathrm{p}}\left[12 \pi\left(D_{3} H\right)^{2}\right],
$$

where $A_{\mathrm{p}}$ is the average surface area of two particles, and $\mathrm{D}_{3} \mathrm{H}$ represents the dimensionless surface-to-surface distance between two particles.

At the critical cogulation contration,

$$
I_{\mathrm{t}}=0 \quad \text { and } \quad f_{\mathrm{t}}=0 .
$$

\section{ACKNOWLEDGMENT}

This work was supported by the National Science Council of the Republic of China under Grant NSC84-2214-E002-030.

\section{REFERENCES}

1. Hunter, R. J., “Foundations of Colloid Science,'” Vol. I. Oxford Univ. Press, London, 1989.

2. Hiemenz, P. C., 'Principles of Colloid and Surface Chemistry,', 2nd ed. Dekker, New York, 1986. 
3. Wiese, G. R., and Healy, T. W., Trans. Faraday Soc. 66, 490 (1970).

4. Metcalfe, I. M., and Healy, T. W., Faraday Discus. Chem. Soc. 90, 335 (1990).

5. Overbeek, J. Th. G., Pure Appl. Chem. 52, 1151 (1980).

6. Donath, E., and Pastushenko, V., Bioelectrochem. Bioenerg. 6, 543 (1979).

7. Wunderlich, R. W., J. Colloid Interface Sci. 88, 385 (1982).

8. Levine, S., Levine, M., Sharp, K. A., and Brooks, D. E., Biophys. J. 42, 127 (1983).

9. Sharp, K. A., and Brooks, D. E., Biophys. J. 47, 563 (1985).

10. Reiss, H., and Bassignana, I. C., J. Membrane Sci. 11, 219 (1982).

11. Selvey, C., and Reiss, H., J. Membrane Sci. 23, 11 (1985).

12. Seaman, G. V., in “The Red Blood Cells"' (D. M. Sergenor, Ed.), Vol. II. Academic Press, New York, 1975.
13. Kawahata, S., Ohshima, H., Muramatsu, N., and Kondo, T., J. Colloid Interface Sci. 138, 182 (1990).

14. Terui, H., Taguchi, T., Ohshima, H., and Kondo, T., Colloid Polym. Sci. 268, 76 (1990).

15. Taguchi, T., Terui, H., Ohshima, H., and Kondo, T., Colloid Polym. Sci. 268, 83 (1990).

16. Hsu, J. P., and Kuo, Y. C., J. Chem. Phys. 103, 465 (1995).

17. Hsu, J. P., and Kuo, Y. C., J. Chem. Soc. Faraday Trans. 91, 1223 (1995).

18. Fuchs, N., Z. Phys. 89, 736 (1934).

19. Overbeek, J. Th. G., in "Colloid Science" (H. R. Kruyt, Ed.), Vol. 1. Elsevier, Amsterdam, 1952.

20. Dukhin, S. S., Semenikhin, N. M., and Shapinskaya, L. M., Dokl. Phys. Chem. 193, 540 (1970). 